\title{
LOWER BOUND OF SECTIONAL CURVATURE OF MANIFOLD OF BETA DISTRIBUTIONS AND COMPLETE MONOTONICITY OF FUNCTIONS INVOLVING POLYGAMMA FUNCTIONS
}

\author{
FENG QI \\ Dedicated to my PhD supervisor, Prof. Sen-Lin Xu, retired at USTC in China \\ on his 80th birthday anniversary \\ Institute of Mathematics, Henan Polytechnic University, Jiaozuo 454010, Henan, China; \\ College of Mathematics, Inner Mongolia University for Nationalities, Tongliao 028043, China; \\ School of Mathematical Sciences, Tianjin Polytechnic University, Tianjin 300387, China \\ E-mail:qqifeng618@gmail.com, qifeng618@hotmail.com, qifeng618@qq.com \\ URL: https: // qifeng618. wordpress. com, https: // orcid. org/0000-0001-6239-2968
}

ABSTRACT. In the paper, by convolution theorem for the Laplace transforms and analytic techniques, the author finds necessary and sufficient conditions for complete monotonicity, monotonicity, and inequalities of several functions involving polygamma functions. By these results, the author derives a lower bound of a function related to the sectional curvature of the manifold of the beta distributions. Finally, the author poses several guesses and open problems related to monotonicity, complete monotonicity, and inequalities of several functions involving polygamma functions.

\section{Contents}

\begin{tabular}{|c|c|}
\hline 1. Motivations & 1 \\
\hline 2. Lemmas & 4 \\
\hline 3. Necessary and sufficient conditions of complete monotonicity & 8 \\
\hline 4. A completely monotonic function involving tetragamma function & 16 \\
\hline 5. The lower bound of sectional curvature & 18 \\
\hline 6. $\quad$ Several remarks, guesses, and open problems & 19 \\
\hline References & 20 \\
\hline
\end{tabular}

\section{Motivations}

In the literature [1, Section 6.4], the function

$$
\Gamma(z)=\int_{0}^{\infty} t^{z-1} e^{-t} \mathrm{~d} t, \quad \Re(z)>0
$$

and its logarithmic derivative $\psi(z)=[\ln \Gamma(z)]^{\prime}=\frac{\Gamma^{\prime}(z)}{\Gamma(z)}$ are respectively called Euler's gamma function and digamma function. Further, the functions $\psi^{\prime}(z)$, $\psi^{\prime \prime}(z), \psi^{\prime \prime \prime}(z)$, and $\psi^{(4)}(z)$ are known as trigamma, tetragamma, pentagamma,

2010 Mathematics Subject Classification. Primary 33B15; Secondary 26A48, 26A51, 26D07, 44A10, 53C25, 60E05, 62H10.

Key words and phrases. necessary and sufficient condition; complete monotonicity; monotonicity; inequality; polygamma function; lower bound; sectional curvature; manifold; beta distribution; convolution theorem for the Laplace transforms and analytic techniques; guess; open problem. This paper was typeset using $\mathcal{A} \mathcal{M} \mathcal{S}$-LATEX. 
and hexagamma functions respectively. As a whole, all the derivatives $\psi^{(k)}(z)$ for $k \in\{0\} \cup \mathbb{N}$ are known as polygamma functions.

Recall from Chapter XIII in [9], Chapter 1 in [21, and Chapter IV in [22] that, if a function $f(x)$ on an interval $I$ has derivatives of all orders on $I$ and satisfies $(-1)^{n} f^{(n)}(x) \geq 0$ for $x \in I$ and $n \in\{0\} \cup \mathbb{N}$, where $\mathbb{N}$ denotes the set of all positive integers, then we call $f(x)$ a completely monotonic function on $I$. Theorem $12 \mathrm{~b}$ in $[22$, p. 161] characterized that a function $f(x)$ is completely monotonic on $(0, \infty)$ if and only if

$$
f(x)=\int_{0}^{\infty} e^{-x t} \mathrm{~d} \sigma(t), \quad x \in(0, \infty),
$$

where $\sigma(s)$ is non-decreasing and the integral in 1.1 converges for $x \in(0, \infty)$. The integral representation (1.1) means that a function $f(x)$ is completely monotonic on $(0, \infty)$ if and only if it is a Laplace transform of a non-decreasing measure $\sigma(s)$ on $(0, \infty)$.

In [7. Proposition 3] and [8, Proposition 13], the sectional curvature $K(x, y)$ of the manifold of the beta distributions was given by

$$
K(x, y)=\frac{1}{4} \frac{\psi^{\prime \prime}(x) \psi^{\prime \prime}(y) \psi^{\prime \prime}(x+y)\left[\frac{\psi^{\prime}(x)}{\psi^{\prime \prime}(x)}+\frac{\psi^{\prime}(y)}{\psi^{\prime \prime}(y)}-\frac{\psi^{\prime}(x+y)}{\psi^{\prime \prime}(x+y)}\right]}{\left[\psi^{\prime}(x) \psi^{\prime}(x+y)+\psi^{\prime}(y) \psi^{\prime}(x+y)-\psi^{\prime}(x) \psi^{\prime}(y)\right]^{2}} .
$$

In [7, Proposition 4] and [8, Proposition 14], the following limits were computed:

$$
\begin{gathered}
\lim _{y \rightarrow 0^{+}} K(x, y)=\lim _{y \rightarrow 0^{+}} K(y, x)=\frac{1}{2}\left(\frac{3}{2}-\frac{\psi^{\prime}(x) \psi^{\prime \prime \prime}(x)}{\left[\psi^{\prime \prime}(x)\right]^{2}}\right), \\
\lim _{y \rightarrow \infty} K(x, y)=\lim _{y \rightarrow \infty} K(y, x)=\frac{1}{4} \frac{\Phi^{\prime}(x)}{\Phi^{2}(x)}, \\
\lim _{(x, y) \rightarrow\left(0^{+}, 0^{+}\right)} K(x, y)=0, \quad \lim _{(x, y) \rightarrow(\infty, \infty)} K(x, y)=-\frac{1}{2}, \\
\lim _{(x, y) \rightarrow\left(0^{+}, \infty\right)} K(x, y)=\lim _{(x, y) \rightarrow\left(\infty, 0^{+}\right)} K(x, y)=-\frac{1}{4},
\end{gathered}
$$

where

$$
\Phi(x)=x \psi^{\prime}(x)-1=x\left[\psi^{\prime}(x)-\frac{1}{x}\right]
$$

on $(0, \infty)$.

In [14, Theorem 4.1] and [16, Theorem 4], the author presented that

(1) if and only if $\alpha \geq 2$, the function $\mathfrak{H}_{\alpha}(x)=\Phi^{\prime}(x)+\alpha \Phi^{2}(x)$ is completely monotonic on $(0, \infty)$

(2) if and only if $\alpha \leq 1$, the function $-\mathfrak{H}_{\alpha}(x)$ is completely monotonic on $(0, \infty)$;

(3) the double inequality $-2<\frac{\Phi^{\prime}(x)}{\Phi^{2}(x)}<-1$ is valid on $(0, \infty)$ and sharp in the sense that the constants -2 and -1 cannot be replaced by any bigger and smaller numbers respectively.

In [14, Theorem 1.1], the author found the following necessary and sufficient conditions and limits:

(1) if and only if $\beta \geq 2$, the function $H_{\beta}(x)=\frac{\Phi^{\prime}(x)}{\Phi^{\beta}(x)}$ is decreasing on $(0, \infty)$, with the limits

$$
\lim _{x \rightarrow 0^{+}} H_{\beta}(x)=\left\{\begin{array}{ll}
-1, & \beta=2 \\
0, & \beta>2
\end{array} \text { and } \quad \lim _{x \rightarrow \infty} H_{\beta}(x)= \begin{cases}-2, & \beta=2 \\
-\infty, & \beta>2\end{cases}\right.
$$


(2) if $\beta \leq 1$, the function $H_{\beta}(x)$ is increasing on $(0, \infty)$, with the limits

$$
H_{\beta}(x) \rightarrow \begin{cases}-\infty, & x \rightarrow 0^{+} \\ 0, & x \rightarrow \infty\end{cases}
$$

In [10], the author considered the functions

$$
\mathfrak{J}_{k, \lambda_{k}}(x)=\Phi^{(2 k+1)}(x)+\lambda_{k}\left[\Phi^{(k)}(x)\right]^{2} \quad \text { and } \quad J_{k, \mu_{k}}(x)=\frac{\Phi^{(2 k+1)}(x)}{\left[(-1)^{k} \Phi^{(k)}(x)\right]^{\mu_{k}}}
$$

on $(0, \infty)$ for $k \in\{0\} \cup \mathbb{N}$ and $\lambda_{k}, \mu_{k} \in \mathbb{R}$. It is clear that $\mathfrak{J}_{0, \lambda_{0}}(x)=\mathfrak{H}_{\lambda_{0}}(x)$ and $J_{0, \mu_{0}}(x)=H_{\mu_{0}}(x)$ for $\lambda_{0}=\alpha$ and $\mu_{0}=\beta$. In [10, Theorems 3.1 and 4.1], the author discovered the following necessary and sufficient conditions, limits, and double inequality:

(1) if and only if $\lambda_{k} \geq \frac{(2 k+2) !}{k !(k+1) !}$, the function $\mathfrak{J}_{k, \lambda_{k}}(x)$ is completely monotonic on $(0, \infty)$;

(2) if and only if $\lambda_{k} \leq \frac{1}{2} \frac{(2 k+2) !}{k !(k+1) !}$, the function $-\mathfrak{J}_{k, \lambda_{k}}(x)$ is completely monotonic on $(0, \infty)$;

(3) if and only if $\mu_{k} \geq 2$, the function $J_{k, \mu_{k}}(x)$ is decreasing on $(0, \infty)$, with the limits

$$
\lim _{x \rightarrow 0^{+}} J_{k, \mu_{k}}(x)= \begin{cases}-\frac{1}{2} \frac{(2 k+2) !}{k !(k+1) !}, & \mu_{k}=2 \\ 0, & \mu_{k}>2\end{cases}
$$

and

$$
\lim _{x \rightarrow \infty} J_{k, \mu_{k}}(x)= \begin{cases}-\frac{(2 k+2) !}{k !(k+1) !}, & \mu_{k}=2 \\ -\infty, & \mu_{k}>2\end{cases}
$$

(4) if $\mu_{k} \leq 1$, the function $J_{k, \mu_{k}}(x)$ is increasing on $(0, \infty)$, with the limits

$$
J_{k, \mu_{k}}(x) \rightarrow \begin{cases}-\infty, & x \rightarrow 0^{+} \\ 0, & x \rightarrow \infty\end{cases}
$$

(5) the double inequality

$$
-\frac{(2 k+2) !}{k !(k+1) !}<\frac{\Phi^{(2 k+1)}(x)}{\left[\Phi^{(k)}(x)\right]^{2}}<-\frac{1}{2} \frac{(2 k+2) !}{k !(k+1) !}
$$

is valid on $(0, \infty)$ and sharp in the sense that the lower and upper bounds cannot be replaced by any larger and smaller numbers respectively.

In the paper [15], the author considered the functions

$$
\begin{gathered}
G(x)=x \Phi(x)-\frac{1}{2}=x\left[x \psi^{\prime}(x)-1\right]-\frac{1}{2}=x^{2}\left[\psi^{\prime}(x)-\frac{1}{x}-\frac{1}{2 x^{2}}\right] \\
\mathcal{G}_{\theta_{k}}(x)=G^{(2 k+1)}(x)+\theta_{k}\left[G^{(k)}(x)\right]^{2}, \quad \mathfrak{G}_{\tau_{k}}(x)=\frac{G^{(2 k+1)}(x)}{\left[(-1)^{k} G^{(k)}(x)\right]^{\tau_{k}}}
\end{gathered}
$$

on $(0, \infty)$ for $k \in\{0\} \cup \mathbb{N}$ and $\theta_{k}, \tau_{k} \in \mathbb{R}$. In [15, Theorems 3.1 and 4.1], the author presented the following necessary and sufficient conditions, limits, and double inequalities:

(1) if and only if $\theta_{k} \geq \frac{3(2 k+2) !}{k !(k+1) !}$, the function $\mathcal{G}_{k, \theta_{k}}(x)$ is completely monotonic on $(0, \infty)$;

(2) if and only if $\theta_{k} \leq 0$, the function $-\mathcal{G}_{k, \theta_{k}}(x)$ is completely monotonic on $(0, \infty)$;

(3) if and only if $\tau_{k} \geq 2$, the function $\mathfrak{G}_{k, \tau_{k}}(x)$ is decreasing on $(0, \infty)$; 
(4) if $\tau_{k} \leq 1$, the function $\mathfrak{G}_{k, \tau_{k}}(x)$ is increasing on $(0, \infty)$;

(5) only if

$$
\tau_{k} \leq \begin{cases}\psi^{\prime}(1), & k=0 \\ -\frac{\psi^{\prime \prime \prime}(1)}{\psi^{\prime}(1) \psi^{\prime \prime}(1)}, & k=1 \\ \frac{k-1}{k} \frac{\psi^{(k-1)}(1) \psi^{(2 k+1)}(1)}{\psi^{(k)}(1) \psi^{(2 k)}(1)}, & k \geq 2\end{cases}
$$

the function $\mathfrak{G}_{k, \tau_{k}}(x)$ is increasing on $(0, \infty)$;

(6) the following two limits are valid:

$$
\lim _{x \rightarrow 0^{+}} \mathfrak{G}_{k, \tau_{k}}(x)= \begin{cases}-2^{\tau_{0}}, & k=0 \\ 6 \psi^{\prime \prime}(1), & k=1 \\ \frac{2(2 k+1)}{(k-1)^{\tau_{k}} k^{\tau_{k}-1}} \frac{\psi^{(2 k)}(1)}{\left|\psi^{(k-1)}(1)\right|}, & k \geq 2\end{cases}
$$

and

$$
\lim _{x \rightarrow \infty} \mathfrak{G}_{k, \tau_{k}}(x)= \begin{cases}-\infty, & \tau_{k}>2 \\ -\frac{3(2 k+2) !}{k !(k+1) !}, & \tau_{k}=2 \\ 0, & \tau_{k}<2\end{cases}
$$

(7) the double inequality

$$
-\frac{3(2 k+2) !}{k !(k+1) !}<\mathfrak{G}_{k, 2}(x)< \begin{cases}-4, & k=0 \\ 6 \psi^{\prime \prime}(1), & k=1 \\ \frac{2(2 k+1)}{(k-1)^{2} k} \frac{\psi^{(2 k)}(1)}{\left|\psi^{(k-1)}(1)\right|}, & k \geq 2\end{cases}
$$

is valid on $(0, \infty)$ and sharp in the sense that the lower and upper bounds cannot be replaced by any greater and less numbers respectively.

In $[7$, Proposition 5], the sectional curvature $K(x, y)$ was proved to be negative and bounded from below. On 19 February 2020, Alice Le Brigant, the first author of the papers $[7,8]$ told the author of this paper via e-mails and the ResearchGate that the lower bound of $K(x, y)$ should be $-\frac{1}{2}$.

Conjecture 1.1 ([8, pp. 12-13]). For $x, y>0$, the sectional curvature $K(x, y)$

(1) has a lower bound $-\frac{1}{2}$, accurately, $K(x, y)>-\frac{1}{2}$;

(2) is decreasing in both $x$ and $y$.

In this paper, we consider the function

$$
\mathcal{K}(x)=K(x, x)=\frac{1}{4} \frac{\psi^{\prime \prime}(x)}{\left[\psi^{\prime}(x)\right]^{2}} \frac{2 \psi^{\prime}(x) \psi^{\prime \prime}(2 x)-\psi^{\prime}(2 x) \psi^{\prime \prime}(x)}{\left[\psi^{\prime}(x)-2 \psi^{\prime}(2 x)\right]^{2}}
$$

on $(0, \infty)$ and prove the sharp double inequality

$$
0>\mathcal{K}(x)>-\frac{1}{2}
$$

which verifies the first conjecture in Conjecture 1.1 along the half-line $x=y>0$ in the first quadrant on $\mathbb{R}^{2}$. 


\section{LEMMAS}

The following lemmas are necessary in this paper.

Lemma 2.1. For $k \in \mathbb{N}$, we have the limits

$$
\lim _{x \rightarrow 0^{+}}\left[x^{k} \psi^{(k-1)}(x)\right]=(-1)^{k}(k-1) !
$$

and

$$
\lim _{x \rightarrow \infty}\left[x^{k} \psi^{(k)}(x)\right]=(-1)^{k-1}(k-1) !
$$

Proof. These two limits can be found in [5, p. 9896, (13)], [11, p. 260, (2.2)], [18 p. $1689,(3.3)]$, [19, p. 286, (2.6)], [20, p. 81, (41)], and [23, p. 769], for example.

Lemma 2.2 (Convolution theorem for the Laplace transforms [22, pp. 91-92]). Let $f_{k}(t)$ for $k=1,2$ be piecewise continuous in arbitrary finite intervals included in $(0, \infty)$. If there exist some constants $M_{k}>0$ and $c_{k} \geq 0$ such that $\left|f_{k}(t)\right| \leq M_{k} e^{c_{k} t}$ for $k=1,2$, then

$$
\int_{0}^{\infty}\left[\int_{0}^{t} f_{1}(u) f_{2}(t-u) \mathrm{d} u\right] e^{-s t} \mathrm{~d} t=\int_{0}^{\infty} f_{1}(u) e^{-s u} \mathrm{~d} u \int_{0}^{\infty} f_{2}(v) e^{-s v} \mathrm{~d} v .
$$

Lemma 2.3. Let

Then the following conclusions are valid:

$$
g(t)= \begin{cases}\frac{t}{1-e^{-t}}, & t \neq 0 \\ 1, & t=0\end{cases}
$$

(1) the function $g(t)$

(a) satisfies the identity

$$
g(t)-g(-t)=t
$$

on $(-\infty, \infty)$;

(b) is infinitely differentiable on $(-\infty, \infty)$, increasing from $(-\infty, \infty)$ onto $(0, \infty)$, convex on $(-\infty, \infty)$, and logarithmically concave on $(-\infty, \infty)$;

(2) the function $\frac{g(2 t)}{g^{2}(t)}$ is increasing from $(-\infty, 0)$ onto $(0,1)$ and decreasing from $(0, \infty)$ onto $(0,1)$;

(3) the double inequality

$$
0<\frac{g(2 t)}{g^{2}(t)}<1
$$

is valid on $(0, \infty)$ and sharp in the sense that the lower bound 0 and the upper bound 1 cannot be replaced by any larger scalar and any smaller scalar respectively;

(4) for any fixed $t>0$, the function $g(s t) g((1-s) t)$ is increasing in $s \in\left(0, \frac{1}{2}\right)$.

Proof. The verification of the identity 2.3 is straightforward.

The differentiability, monotonicity, and convexity of $g(t)$ come from utilization of [14, Lemma 2.3].

Direct computation yields

$$
\begin{aligned}
& {[\ln g(t)]^{\prime \prime}=[\ln g(-t)]^{\prime \prime}=-\frac{e^{2 t}-e^{t}\left(t^{2}+2\right)+1}{\left(e^{t}-1\right)^{2} t^{2}}} \\
& =-\frac{1}{\left(e^{t}-1\right)^{2} t^{2}} \sum_{k=4}^{\infty}\left[2^{k}-(k-1) k-2\right] \frac{t^{k}}{k !}<0
\end{aligned}
$$

on $(0, \infty)$. Hence, the function $g(t)$ is logarithmically concave on $(-\infty, \infty)$. 
Standard calculation shows

$$
\frac{g(2 t)}{g^{2}(t)}=\frac{2\left(e^{t}-1\right)}{\left(e^{t}+1\right) t} \rightarrow \begin{cases}1, & t \rightarrow 0 \\ 0, & t \rightarrow \pm \infty\end{cases}
$$

and

$$
\begin{gathered}
{\left[\frac{g(2 t)}{g^{2}(t)}\right]^{\prime}=\left[\frac{g(-2 t)}{g^{2}(-t)}\right]^{\prime}=-\frac{2\left(e^{2 t}-2 t e^{t}-1\right)}{\left(e^{t}+1\right)^{2} t^{2}}} \\
=-\frac{4}{\left(e^{t}+1\right)^{2} t^{2}} \sum_{k=3}^{\infty} \frac{\left(2^{k-1}-k\right) t^{k}}{k !}<0
\end{gathered}
$$

on $(0, \infty)$. This implies the monotonicity of $\frac{g(2 t)}{g^{2}(t)}$ and leads to the double inequality 2.4 and its sharpness.

Direct differentiation gives

$$
\begin{aligned}
\frac{\mathrm{d}[g(s t) g((1-s) t)]}{\mathrm{d} s} & =t g^{\prime}(s t) g((1-s) t)-t g(s t) g^{\prime}((1-s) t) \\
& =\operatorname{tg}(s t) g((1-s) t)\left[\frac{g^{\prime}(s t)}{g(s t)}-\frac{g^{\prime}((1-s) t)}{g((1-s) t)}\right] \\
& =t g(s t) g((1-s) t)\left[\left.\frac{\mathrm{d} \ln g(s)}{\mathrm{d} s}\right|_{s=s t}-\left.\frac{\mathrm{d} \ln g(s)}{\mathrm{d} s}\right|_{s=(1-s) t}\right] \\
& >0
\end{aligned}
$$

for $0<s<\frac{1}{2}$, where we used in the last step the facts that $s t<(1-s) t$ for $0<s<\frac{1}{2}$ and that $g(t)$ is logarithmically concave on $(-\infty, \infty)$. Accordingly, for any fixed $t>0$, the function $g(s t) g((1-s) t)$ is increasing in $s \in\left(0, \frac{1}{2}\right)$. The proof of Lemma 2.3 is complete.

Lemma 2.4 ([12, Theorem 6.1]). If $f(x)$ is differentiable and logarithmically concave on $(-\infty, \infty)$, then the product $f(x) f\left(x_{0}-x\right)$ for any fixed number $x_{0} \in \mathbb{R}$ is increasing in $x \in\left(-\infty, \frac{x_{0}}{2}\right)$ and decreasing in $x \in\left(\frac{x_{0}}{2}, \infty\right)$.

Lemma 2.5 ([10, Lemma 2.2]). For $k \geq 0$, we have

$$
(-1)^{k} x^{k+1} \Phi^{(k)}(x) \rightarrow \begin{cases}k !, & x \rightarrow 0^{+} \\ \frac{k !}{2}, & x \rightarrow \infty\end{cases}
$$

Lemma 2.6 ([10, Lemma 2.6]). For $k, m \in \mathbb{N}$, the function

$$
V_{k, m}(x)=\frac{(1-x)^{k+m}+(1+x)^{k+m}}{(1-x)^{k}+(1+x)^{k}}
$$

is increasing in $x \in[0,1]$, with $V_{k, m}(0)=1$ and $V_{k, m}(1)=2^{m}$.

Lemma 2.7. For $t>u>0$, the function

$$
W_{t}(u)=\frac{\left[e^{-(t-u) / 2}-e^{-u / 2}\right](t-u)}{\left(1-e^{-u / 2}\right)\left[1-e^{-(t-u) / 2}\right]}
$$

is increasing in $u \in(0, t)$, with limits

$$
\lim _{u \rightarrow t^{-}} W_{t}(u)=2 \quad \text { and } \quad \lim _{u \rightarrow 0^{+}} W_{t}(u)=-\infty .
$$

Proof. It is easy to see that

$$
\lim _{u \rightarrow t^{-}} W_{t}(u)=\frac{\lim _{u \rightarrow t^{-}}\left[e^{-(t-u) / 2}-e^{-u / 2}\right]}{1-e^{-u / 2}} \lim _{u \rightarrow t^{-}} \frac{t-u}{1-e^{-(t-u) / 2}}=2 .
$$


Direct differentiation yields

$$
\begin{aligned}
& \frac{\mathrm{d} W(2 t, 2 u)}{\mathrm{d} u}=\frac{2\left[\begin{array}{c}
e^{4 u}+\left(e^{t}+1\right)(t-u-1) e^{3 u}-4(t-u) e^{t+2 u} \\
+(t-u+1) e^{t+u}+(t-u+1) e^{2 t+u}-e^{2 t}
\end{array}\right]}{\left(e^{u}-1\right)^{2}\left(e^{t}-e^{u}\right)^{2}} \\
& \triangleq \frac{2 W_{1}(t, u)}{\left(e^{u}-1\right)^{2}\left(e^{t}-e^{u}\right)^{2}}, \\
& \lim _{u \rightarrow t^{-}} W_{1}(t, u)=0 \\
& \frac{\mathrm{d} W_{1}(t, u)}{\mathrm{d} u}=e^{u}\left[4 e^{3 u}+(3 t-3 u-4) e^{2 u}\left(1+e^{t}\right)\right. \\
& \left.-4(2 t-2 u-1) e^{t+u}+(t-u)\left(1+e^{t}\right) e^{t}\right] \\
& \triangleq e^{u} W_{2}(t, u) \\
& \rightarrow 0, \quad u \rightarrow t^{-}, \\
& \frac{\mathrm{d} W_{2}(t, u)}{\mathrm{d} u}=12 e^{3 u}+(6 t-6 u-11)\left(1+e^{t}\right) e^{2 u} \\
& -4(2 t-2 u-3) e^{t+u}-e^{t}\left(1+e^{t}\right) \\
& \rightarrow\left(e^{2 t}-1\right) e^{t} \\
& >0 \\
& \frac{\mathrm{d}^{2} W_{2}(t, u)}{\mathrm{d} u^{2}}=4 e^{u}\left[9 e^{2 u}+(3 t-3 u-7)\left(1+e^{t}\right) e^{u}-e^{t}(2 t-2 u-5)\right] \\
& \triangleq 4 e^{u} W_{3}(t, u) \\
& \rightarrow 8 e^{2 t}\left(e^{t}-1\right), \quad u \rightarrow t^{-} \\
& >0 \\
& \frac{\mathrm{d} W_{3}(t, u)}{\mathrm{d} u}=18 e^{2 u}+(3 t-3 u-10)\left(1+e^{t}\right) e^{u}+2 e^{t} \\
& \rightarrow 8 e^{t}\left(e^{t}-1\right), u \rightarrow t^{-} \\
& >0 \\
& \frac{\mathrm{d}^{2} W_{3}(t, u)}{\mathrm{d} u^{2}}=e^{u}\left[36 e^{u}+(3 t-3 u-13) e^{t}+3 t-3 u-13\right] \\
& \triangleq e^{u} W_{4}(t, u) \\
& \rightarrow e^{t}\left(23 e^{t}-13\right), \quad u \rightarrow t^{-} \\
& >0 \text {, } \\
& \frac{\mathrm{d} W_{4}(t, u)}{\mathrm{d} u}=3\left(12 e^{u}-e^{t}-1\right) \\
& \rightarrow 3\left(11 e^{t}-1\right), \quad u \rightarrow t^{-} \\
& >0 \text {, } \\
& \begin{aligned}
\frac{\mathrm{d}^{2} W_{4}(t, u)}{\mathrm{d} u^{2}} & =36 e^{u} \\
& >0
\end{aligned}
\end{aligned}
$$

for $t>0$ and $u \in(0, t)$. Accordingly, all the functions $W_{\ell}(t, u)$ for $2 \leq \ell \leq 4$ are positive, increasing, and convex in $u \in(0, t)$. Therefore, the function $W_{1}(t, u)$ is positive and increasing in $u \in(0, t)$. Hence, the function $W_{t}(u)$ is increasing in $u \in(0, t)$. As a result, the inequality $W_{t}(u)<2$ is sharp for $t>0$ and $u \in(0, t)$. The proof of Lemma 2.7 is complete. 
Lemma 2.8. For $k \in\{0\} \cup \mathbb{N}$ and $a \geq 0$, we have

$$
\lim _{x \rightarrow \infty}\left(x^{k+1}\left[\psi^{(k)}(x+a)-\psi^{(k)}(x)\right]\right)=(-1)^{k} k ! a .
$$

For $k, \ell \in \mathbb{N}$ and $a \geq 0$, we have

$$
\begin{aligned}
\lim _{x \rightarrow \infty}\left(x ^ { k + \ell + 1 } \left[\psi^{(k)}(x) \psi^{(\ell+1)}(x)-\psi^{(k+1)}\right.\right. & \left.\left.(x) \psi^{(\ell)}(x)\right]\right) \\
& =(-1)^{k+\ell}(k-1) !(\ell-1) !(k-\ell)
\end{aligned}
$$

and

$$
\begin{aligned}
\lim _{x \rightarrow \infty}\left(x ^ { k + \ell + 1 } \left[\psi^{(k)}(x) \psi^{(\ell)}(x+a)-\psi^{(\ell)}\right.\right. & \left.\left.(x) \psi^{(k)}(x+a)\right]\right) \\
& =(-1)^{k+\ell}(k-1) !(\ell-1) !(k-\ell) a .
\end{aligned}
$$

Proof. It is straightforward that

$$
\begin{aligned}
& \lim _{x \rightarrow \infty}\left(x^{k+1}\left[\psi^{(k)}(x+a)-\psi^{(k)}(x)\right]\right) \\
= & \lim _{x \rightarrow \infty}\left[x^{k+1} \int_{x}^{x+a} \psi^{(k+1)}(u) \mathrm{d} u\right] \\
= & \lim _{x \rightarrow \infty}\left[x^{k+1} \int_{0}^{a} \psi^{(k+1)}(x+u) \mathrm{d} u\right] \\
= & \int_{0}^{a} \lim _{x \rightarrow \infty}\left[x^{k+1} \psi^{(k+1)}(x+u)\right] \mathrm{d} u \\
= & \int_{0}^{a} \lim _{x \rightarrow \infty}\left[(x+u)^{k+1} \psi^{(k+1)}(x+u)\right] \lim _{x \rightarrow \infty} \frac{x^{k+1}}{(x+u)^{k+1}} \mathrm{~d} u \\
= & \int_{0}^{a}(-1)^{k} k ! \mathrm{d} u \\
= & (-1)^{k} k ! a,
\end{aligned}
$$

where we used the limit 2.2 .

It is also straightforward that

$$
\begin{aligned}
& \lim _{x \rightarrow \infty}\left(x^{k+\ell+1}\left[\psi^{(k)}(x) \psi^{(\ell+1)}(x)-\psi^{(k+1)}(x) \psi^{(\ell)}(x)\right]\right) \\
= & \lim _{x \rightarrow \infty}\left(\left[x^{k} \psi^{(k)}(x)\right]\left[x^{\ell+1} \psi^{(\ell+1)}(x)\right]-\left[x^{k+1} \psi^{(k+1)}(x)\right]\left[x^{\ell} \psi^{(\ell)}(x)\right]\right) \\
= & (-1)^{k-1}(k-1) !(-1)^{\ell} \ell !-(-1)^{k} k !(-1)^{\ell-1}(\ell-1) ! \\
= & (-1)^{k+\ell-1}[(k-1) ! \ell !-k !(\ell-1) !] \\
= & (-1)^{k+\ell}(k-1) !(\ell-1) !(k-\ell)
\end{aligned}
$$

and

$$
\begin{aligned}
& \lim _{x \rightarrow \infty}\left(x^{k+\ell+1}\left[\psi^{(k)}(x) \psi^{(\ell)}(x+a)-\psi^{(\ell)}(x) \psi^{(k)}(x+a)\right]\right) \\
= & \lim _{x \rightarrow \infty}\left(x^{k+\ell+1} \psi^{(k)}(x) \psi^{(k)}(x+a)\left[\frac{\psi^{(\ell)}(x+a)}{\psi^{(k)}(x+a)}-\frac{\psi^{(\ell)}(x)}{\psi^{(k)}(x)}\right]\right) \\
= & \lim _{x \rightarrow \infty}\left(\frac{x^{\ell+1}}{(x+a)^{k}}\left[x^{k} \psi^{(k)}(x)\right]\left[(x+a)^{k} \psi^{(k)}(x+a)\right] \int_{x}^{x+a}\left[\frac{\psi^{(\ell)}(u)}{\psi^{(k)}(u)}\right]^{\prime} \mathrm{d} u\right) \\
= & \lim _{x \rightarrow \infty}\left[x^{k} \psi^{(k)}(x)\right] \lim _{x \rightarrow \infty}\left[(x+a)^{k} \psi^{(k)}(x+a)\right] \\
& \times \lim _{x \rightarrow \infty}\left[\frac{x^{\ell+1}}{(x+a)^{k}} \int_{0}^{a} \frac{\psi^{(\ell+1)}(x+u) \psi^{(k)}(x+u)-\psi^{(\ell)}(x+u) \psi^{(k+1)}(x+u)}{\left[\psi^{(k)}(x+u)\right]^{2}} \mathrm{~d} u\right] \\
= & {[(k-1) !]^{2} \int_{0}^{a} \lim _{x \rightarrow \infty}\left[\frac{x^{\ell+1}}{(x+a)^{k}} \frac{(x+u)^{2 k}}{x^{k+\ell+1}}\right] }
\end{aligned}
$$




$$
\begin{aligned}
& \times \lim _{x \rightarrow \infty} \frac{x^{k+\ell+1}\left[\psi^{(\ell+1)}(x+u) \psi^{(k)}(x+u)-\psi^{(\ell)}(x+u) \psi^{(k+1)}(x+u)\right]}{\left[(x+u)^{k} \psi^{(k)}(x+u)\right]^{2}} \mathrm{~d} u \\
= & (-1)^{k+\ell}(k-1) !(\ell-1) !(k-\ell) a,
\end{aligned}
$$

where we used the limits $(2.2)$ and $(2.7)$. The proof of Lemma 2.8 is complete.

\section{NECESSARY AND SUFFICIENT CONDITIONS OF COMPLETE MONOTONICITY}

For verifying the lower bound in the double inequality (1.3), we find a lower bound for the second factor in 1.2 and more.

Theorem 3.1. Let $p>m \geq n>q \geq 0$ be integers such that $m+n=p+q$ and let

$$
F_{p, m, n, q ; c_{p, m, n, q}}(x)= \begin{cases}\left|\psi^{(m)}(x)\right|\left|\psi^{(n)}(x)\right|-c_{p, m, n, q}\left|\psi^{(p)}(x)\right|, & q=0 \\ \left|\psi^{(m)}(x)\right|\left|\psi^{(n)}(x)\right|-c_{p, m, n, q}\left|\psi^{(p)}(x)\right|\left|\psi^{(q)}(x)\right|, & q \geq 1\end{cases}
$$

for $c_{p, m, n, q} \in \mathbb{R}$ and $x \in(0, \infty)$. Then

(1) for $q \geq 0$, if and only if

$$
c_{p, m, n, q} \leq \begin{cases}\frac{(m-1) !(n-1) !}{(p-1) !}, & q=0 \\ \frac{(m-1) !(n-1) !}{(p-1) !(q-1) !}, & q \geq 1,\end{cases}
$$

the function $F_{p, m, n, q ; c_{p, m, n, q}}(x)$ is completely monotonic in $x \in(0, \infty)$;

(2) for $q \geq 1$, if and only if $c_{p, m, n, q} \geq \frac{m ! n !}{p ! q !}$, the function $-F_{p, m, n, q ; c_{p, m, n, q}}(x)$ is completely monotonic in $x \in(0, \infty)$;

(3) the double inequality

$$
-\frac{(m+n-1) !}{(m-1) !(n-1) !}<\frac{\psi^{(m+n)}(x)}{\psi^{(m)}(x) \psi^{(n)}(x)}<0
$$

for $m, n \in \mathbb{N}$ and the double inequality

$$
\frac{(m-1) !(n-1) !}{(p-1) !(q-1) !}<\frac{\psi^{(m)}(x) \psi^{(n)}(x)}{\psi^{(p)}(x) \psi^{(q)}(x)}<\frac{m ! n !}{p ! q !}
$$

for $m, n, p, q \in \mathbb{N}$ with $p>m \geq n>q \geq 1$ and $m+n=p+q$ are valid on $(0, \infty)$ and sharp in the sense that the lower and upper bounds cannot be replaced by any larger and smaller scalars respectively.

Proof. The sufficient conditions were proved in [3. Theorem 4.1].

The first derivative of the function $F_{p, m, n, q ; c_{p, m, n, q}}(x)$ is

$$
\begin{aligned}
\frac{\partial F_{p, m, n, 0 ; c_{p, m, n, 0}}(x)}{\partial x}= & (-1)^{m+n}\left[\psi^{(m+1)}(x) \psi^{(n)}(x)+\psi^{(m)}(x) \psi^{(n+1)}(x)\right] \\
& -c_{p, m, n, 0}(-1)^{p+1} \psi^{(p+1)}(x)
\end{aligned}
$$

and, for $q \geq 1$,

$$
\begin{aligned}
\frac{\partial F_{p, m, n, q ; c_{p, m, n, q}}(x)}{\partial x} & =(-1)^{m+n}\left[\psi^{(m+1)}(x) \psi^{(n)}(x)+\psi^{(m)}(x) \psi^{(n+1)}(x)\right] \\
& -c_{p, m, n, q}(-1)^{p+q}\left[\psi^{(p+1)}(x) \psi^{(q)}(x)+\psi^{(p)}(x) \psi^{(q+1)}(x)\right] .
\end{aligned}
$$

If $\pm F_{p, m, n, q ; c_{p, m, n, q}}(x)$ is completely monotonic, then $\pm F_{p, m, n, q}^{\prime}\left(x ; c_{p, m, n, q}\right) \leq 0$ which are equivalent to

$c_{p, m, n, 0} \lesseqgtr \frac{(-1)^{m+n}\left[\psi^{(m+1)}(x) \psi^{(n)}(x)+\psi^{(m)}(x) \psi^{(n+1)}(x)\right]}{(-1)^{p+1} \psi^{(p+1)}(x)}$ 


$$
\begin{aligned}
& =\left\{\begin{array}{l}
\frac{x^{m+2} \psi^{(m+1)}(x) x^{n+1} \psi^{(n)}(x)+x^{m+1} \psi^{(m)}(x) x^{n+2} \psi^{(n+1)}(x)}{x^{m+n+3-p-2}(-1)^{m+n+p+1} x^{p+2} \psi^{(p+1)}(x)} \\
\frac{x^{m+1} \psi^{(m+1)}(x) x^{n} \psi^{(n)}(x)+x^{m} \psi^{(m)}(x) x^{n+1} \psi^{(n+1)}(x)}{x^{m+n+1-p-1}(-1)^{m+n+p+1} x^{p+1} \psi^{(p+1)}(x)}
\end{array}\right. \\
& =\left\{\begin{array}{l}
\frac{x^{m+2} \psi^{(m+1)}(x) x^{n+1} \psi^{(n)}(x)+x^{m+1} \psi^{(m)}(x) x^{n+2} \psi^{(n+1)}(x)}{(-x)\left[x^{p+2} \psi^{(p+1)}(x)\right]} \\
\frac{x^{m+1} \psi^{(m+1)}(x) x^{n} \psi^{(n)}(x)+x^{m} \psi^{(m)}(x) x^{n+1} \psi^{(n+1)}(x)}{-x^{p+1} \psi^{(p+1)}(x)}
\end{array}\right. \\
& \rightarrow \begin{cases}\frac{(-1)^{m+2}(m+1) !(-1)^{n+1} n !+(-1)^{m+1} m !(-1)^{n+2}(n+1) !}{-\left(\lim _{x \rightarrow 0^{+}} x\right)\left[(-1)^{p+2}(p+1) !\right]}, & x \rightarrow 0^{+} \\
\frac{(-1)^{m} m !(-1)^{n-1}(n-1) !+(-1)^{m-1}(m-1) !(-1)^{n} n !}{-(-1)^{p} p !}, & x \rightarrow \infty\end{cases} \\
& =\left\{\begin{array}{l}
\infty \\
\frac{m !(n-1) !+(m-1) ! n !}{p !}
\end{array}\right. \\
& =\left\{\begin{array}{l}
\infty \\
\frac{(m-1) !(n-1) !}{(p-1) !}
\end{array}\right.
\end{aligned}
$$

and

$$
\begin{aligned}
& c_{p, m, n, q} \lesseqgtr \frac{(-1)^{m+n}\left[\psi^{(m+1)}(x) \psi^{(n)}(x)+\psi^{(m)}(x) \psi^{(n+1)}(x)\right]}{(-1)^{p+q}\left[\psi^{(p+1)}(x) \psi^{(q)}(x)+\psi^{(p)}(x) \psi^{(q+1)}(x)\right]} \\
& =\left\{\begin{array}{l}
\frac{x^{m+2} \psi^{(m+1)}(x) x^{n+1} \psi^{(n)}(x)+x^{m+1} \psi^{(m)}(x) x^{n+2} \psi^{(n+1)}(x)}{x^{p+2} \psi^{(p+1)}(x) x^{q+1} \psi^{(q)}(x)+x^{p+1} \psi^{(p)}(x) x^{q+2} \psi^{(q+1)}(x)} \\
\frac{x^{m+1} \psi^{(m+1)}(x) x^{n} \psi^{(n)}(x)+x^{m} \psi^{(m)}(x) x^{n+1} \psi^{(n+1)}(x)}{x^{p+1} \psi^{(p+1)}(x) x^{q} \psi^{(q)}(x)+x^{p} \psi^{(p)}(x) x^{q+1} \psi^{(q+1)}(x)}
\end{array}\right. \\
& \rightarrow \begin{cases}\frac{(-1)^{m+2}(m+1) !(-1)^{n+1} n !+(-1)^{m+1} m !(-1)^{n+2}(n+1) !}{(-1)^{p+2}(p+1) !(-1)^{q+1} q !+(-1)^{p+1} p !(-1)^{q+2}(q+1) !}, & x \rightarrow 0^{+} \\
\frac{(-1)^{m} m !(-1)^{n-1}(n-1) !+(-1)^{m-1}(m-1) !(-1)^{n} n !}{(-1)^{p} p !(-1)^{q-1}(q-1) !+(-1)^{p-1}(p-1) !(-1)^{q} q !}, & x \rightarrow \infty\end{cases} \\
& =\left\{\begin{array}{l}
\frac{(m+1) ! n !+m !(n+1) !}{(p+1) ! q !+p !(q+1) !} \\
\frac{m !(n-1) !+(m-1) ! n !}{p !(q-1) !+(p-1) ! q !}
\end{array}\right. \\
& =\left\{\begin{array}{l}
\frac{m ! n !}{p ! q !} \\
\frac{(m-1) !(n-1) !}{(p-1) !(q-1) !}
\end{array}\right.
\end{aligned}
$$

for $m+n=p+q$, where we used the limits 2.1 and (2.2) in Lemma2.1. Moreover, for $p>m \geq n>q>0$ such that $m+n=p+q$, we have

$$
\frac{p / m-1}{n / q-1}=\frac{q}{m}<1 \Longleftrightarrow \frac{m n}{p q}>1 \Longleftrightarrow \frac{m ! n !}{p ! q !}>\frac{(m-1) !(n-1) !}{(p-1) !(q-1) !}
$$

Hence, necessary conditions are proved. 
The double inequalities 3.1 and 3.2 come from the positivity of the functions $\pm F_{p, m, n, q ; c_{p, m, n, q}}(x)$ and their sharpness can be concluded from the limits

$$
\begin{gathered}
\lim _{x \rightarrow 0^{+}} \frac{\psi^{(m+n)}(x)}{\psi^{(m)}(x) \psi^{(n)}(x)}=\lim _{x \rightarrow 0^{+}} \frac{x^{m+n+1} \psi^{(m+n)}(x)}{x^{m+1} \psi^{(m)}(x) x^{n+1} \psi^{(n)}(x)} \lim _{x \rightarrow 0^{+}} x=0, \\
\lim _{x \rightarrow \infty} \frac{\psi^{(m+n)}(x)}{\psi^{(m)}(x) \psi^{(n)}(x)}=\lim _{x \rightarrow \infty} \frac{x^{m+n} \psi^{(m+n)}(x)}{x^{m} \psi^{(m)}(x) x^{n} \psi^{(n)}(x)}=-\frac{(m+n-1) !}{(m-1) !(n-1) !}, \\
\lim _{x \rightarrow 0^{+}} \frac{\psi^{(m)}(x) \psi^{(n)}(x)}{\psi^{(p)}(x) \psi^{(q)}(x)}=\lim _{x \rightarrow 0^{+}} \frac{x^{m+1} \psi^{(m)}(x) x^{n+1} \psi^{(n)}(x)}{x^{p+1} \psi^{(p)}(x) x^{q+1} \psi^{(q)}(x)}=\frac{m ! n !}{p ! q !}, \\
\lim _{x \rightarrow \infty} \frac{\psi^{(m)}(x) \psi^{(n)}(x)}{\psi^{(p)}(x) \psi^{(q)}(x)}=\lim _{x \rightarrow \infty} \frac{x^{m} \psi^{(m)}(x) x^{n} \psi^{(n)}(x)}{x^{p} \psi^{(p)}(x) x^{q} \psi^{(q)}(x)}=\frac{(m-1) !(n-1) !}{(p-1) !(q-1) !}
\end{gathered}
$$

where we used the limits (2.1) and (2.2) in Lemma 2.1 once again. The proof of Theorem 3.1 is complete.

Theorem 3.2. For $k \in \mathbb{N}$ and $x \in(0, \infty)$, let

$$
\mathcal{F}_{k, \eta_{k}}(x)=\psi^{(2 k)}(x)+\eta_{k}\left[\psi^{(k)}(x)\right]^{2} \quad \text { and } \quad \mathfrak{F}_{k, \vartheta_{k}}(x)=\frac{\psi^{(2 k)}(x)}{\left[(-1)^{k+1} \psi^{(k)}(x)\right]^{\vartheta_{k}}} .
$$

Then the following conclusions are true:

(1) if and only if $\eta_{k} \geq \frac{1}{2} \frac{(2 k) !}{(k-1) ! k !}$, the function $\mathcal{F}_{k, \eta_{k}}(x)$ is completely monotonic on $(0, \infty)$;

(2) if and only if $\eta_{k} \leq 0$, the function $-\mathcal{F}_{k, \eta_{k}}(x)$ is completely monotonic on $(0, \infty)$;

(3) if and only if $\vartheta_{k} \geq 2$, the function $\mathfrak{F}_{k, \vartheta_{k}}(x)$ is decreasing on $(0, \infty)$;

(4) if and only if $\vartheta_{k} \leq \frac{2 k+1}{k+1}$, the function $\mathfrak{F}_{k, \vartheta_{k}}(x)$ is increasing on $(0, \infty)$;

(5) the following limits are valid:

$$
\lim _{x \rightarrow 0^{+}} \mathfrak{F}_{k, \vartheta_{k}}(x)= \begin{cases}-\frac{(2 k) !}{[(k) !]^{\frac{2 k+1}{k+1}}}, & \vartheta_{k}=\frac{2 k+1}{k+1} \\ 0, & \vartheta_{k}>\frac{2 k+1}{k+1} \\ -\infty, & \vartheta_{k}<\frac{2 k+1}{k+1}\end{cases}
$$

and

$$
\lim _{x \rightarrow \infty} \mathfrak{F}_{k, \vartheta_{k}}(x)= \begin{cases}-\frac{(2 k-1) !}{[(k-1) !]^{2}}, & \vartheta_{k}=2 \\ -\infty, & \vartheta_{k}>2 \\ 0, & \vartheta_{k}<2\end{cases}
$$

(6) the double inequality

$$
-\frac{1}{2} \frac{(2 k) !}{(k-1) ! k !}<\frac{\psi^{(2 k)}(x)}{\left[(-1)^{k+1} \psi^{(k)}(x)\right]^{2}}<0
$$

is valid on $(0, \infty)$ and sharp in the sense that the lower and upper bounds cannot be replaced by any greater and less numbers respectively.

Proof. Taking $q=0, m=n=k$, and $p=2 k$ in Theorem 3.1 leads to that the function

$$
\left[\psi^{(k)}(x)\right]^{2}+c_{2 k, k, k, 0} \psi^{(2 k)}(x)
$$

is completely monotonic on $(0, \infty)$ if and only if $c_{2 k, k, k, 0} \leq \frac{[(k-1) !]^{2}}{(2 k-1) !}$. This result is equivalent to that the function $\mathcal{F}_{k, \eta_{k}}(x)$ is completely monotonic on $(0, \infty)$ if and 
only if $\eta_{k} \geq \frac{(2 k-1) !}{[(k-1) !]^{2}}=\frac{1}{2} \frac{(2 k) !}{(k-1) ! k !}$. For completeness, in what follows, we will prove this result in details once again.

If $\mathcal{F}_{k, \eta_{k}}(x)$ is completely monotonic on $(0, \infty)$, then its first derivative

$$
\left[\mathcal{F}_{k, \eta_{k}}(x)\right]^{\prime}=\psi^{(2 k+1)}(x)+2 \eta_{k} \psi^{(k)}(x) \psi^{(k+1)}(x) \leq 0
$$

which can be rewritten as

$$
\begin{gathered}
\eta_{k} \geq-\frac{1}{2} \frac{\psi^{(2 k+1)}(x)}{\psi^{(k)}(x) \psi^{(k+1)}(x)}=-\frac{1}{2} \frac{x^{2 k+1} \psi^{(2 k+1)}(x)}{\left[x^{k} \psi^{(k)}(x)\right]\left[x^{k+1} \psi^{(k+1)}(x)\right]} \\
\rightarrow-\frac{1}{2} \frac{(-1)^{2 k}(2 k) !}{(-1)^{k-1}(k-1) !(-1)^{k} k !}=\frac{1}{2} \frac{(2 k) !}{(k-1) ! k !}
\end{gathered}
$$

as $x \rightarrow \infty$, where we used the limit (2.2).

If $-\mathcal{F}_{k, \eta_{k}}(x)$ is completely monotonic on $(0, \infty)$, then its first derivative

$$
\eta_{k} \leq-\frac{1}{2} \frac{\psi^{(2 k+1)}(x)}{\psi^{(k)}(x) \psi^{(k+1)}(x)}=-\frac{1}{2} \frac{x\left[x^{2 k+2} \psi^{(2 k+1)}(x)\right]}{\left[x^{k+1} \psi^{(k)}(x)\right]\left[x^{k+2} \psi^{(k+1)}(x)\right]} \rightarrow 0
$$

as $x \rightarrow 0^{+}$, where we used the limit (2.1).

From the integral representation

$$
\psi^{(n)}(z)=(-1)^{n+1} \int_{0}^{\infty} \frac{t^{n}}{1-e^{-t}} e^{-z t} \mathrm{~d} t
$$

for $\Re(z)>0$ and $n \in \mathbb{N}$, see $[1$, p. $260,6.4 .1]$, it follows that $(-1)^{k+1} \psi^{(k)}(x)$ for all $k \in \mathbb{N}$ are completely monotonic on $(0, \infty)$. Further considering the fact that the sum of finite completely monotonic functions is also completely monotonic, we see that the necessary condition $\eta_{k} \leq 0$ is also sufficient for $-\mathcal{F}_{k, \eta_{k}}(x)$ to be completely monotonic on $(0, \infty)$.

By Lemma 2.2 and the integral representation 3.4, we obtain

$$
\begin{aligned}
& \mathcal{F}_{k, \eta_{k}}(x)=\eta_{k}\left[\int_{0}^{\infty} t^{k-1} g(t) e^{-x t} \mathrm{~d} t\right]^{2}-\int_{0}^{\infty} t^{2 k-1} g(t) e^{-x t} \mathrm{~d} t \\
= & \eta_{k} \int_{0}^{\infty}\left[\int_{0}^{t} u^{k-1}(t-u)^{k-1} g(u) g(t-u) \mathrm{d} u\right] e^{-x t} \mathrm{~d} t-\int_{0}^{\infty} t^{2 k-1} g(t) e^{-x t} \mathrm{~d} t \\
= & \int_{0}^{\infty}\left[\eta_{k} \int_{0}^{t} u^{k-1}(t-u)^{k-1} g(u) g(t-u) \mathrm{d} u-t^{2 k-1} g(t)\right] e^{-x t} \mathrm{~d} t,
\end{aligned}
$$

where, by logarithmic convexity of $g(t)$ in Lemma 2.3 and by Lemma 2.4 .

$$
\begin{aligned}
\int_{0}^{t} u^{k-1}(t-u)^{k-1} g(u) g(t-u) \mathrm{d} u & \leq g^{2}\left(\frac{t}{2}\right) \int_{0}^{t} u^{k-1}(t-u)^{k-1} \mathrm{~d} u \\
& =g^{2}\left(\frac{t}{2}\right) t^{2 k-1} \int_{0}^{1} s^{k-1}(1-s)^{k-1} \mathrm{~d} s \\
& =g^{2}\left(\frac{t}{2}\right) t^{2 k-1} B(k, k) \\
& =\frac{[(k-1) !]^{2}}{(2 k-1) !} t^{2 k-1} g^{2}\left(\frac{t}{2}\right)
\end{aligned}
$$

and

$$
\begin{aligned}
\int_{0}^{t} u^{k-1}(t-u)^{k-1} g(u) g(t-u) \mathrm{d} u & \geq g(0) g(t) \int_{0}^{t} u^{k-1}(t-u)^{k-1} \mathrm{~d} u \\
& =\frac{[(k-1) !]^{2}}{(2 k-1) !} t^{2 k-1} g(t) .
\end{aligned}
$$

Accordingly, we acquire 


$$
\begin{aligned}
\eta_{k} \int_{0}^{t} u^{k-1}(t-u)^{k-1} g(u) g(t-u) \mathrm{d} u & -t^{2 k-1} g(t) \\
\leq & {\left[\eta_{k} \frac{[(k-1) !]^{2}}{(2 k-1) !}-\frac{g(t)}{g^{2}\left(\frac{t}{2}\right)}\right] t^{2 k-1} g^{2}\left(\frac{t}{2}\right) }
\end{aligned}
$$

and

$$
\eta_{k} \int_{0}^{t} u^{k-1}(t-u)^{k-1} g(u) g(t-u) \mathrm{d} u-t^{2 k-1} g(t) \geq\left(\eta_{k} \frac{[(k-1) !]^{2}}{(2 k-1) !}-1\right) t^{2 k-1} g(t) .
$$

Therefore, the sharp double inequality (2.4) implies that

$$
\eta_{k} \int_{0}^{t} u^{k-1}(t-u)^{k-1} g(u) g(t-u) \mathrm{d} u-t^{2 k-1} g(t) \leq 0
$$

on $(0, \infty)$ only if $\eta_{k} \leq 0$, while

$$
\eta_{k} \int_{0}^{t} u^{k-1}(t-u)^{k-1} g(u) g(t-u) \mathrm{d} u-t^{2 k-1} g(t) \geq 0
$$

on $(0, \infty)$ only if $\eta_{k} \geq \frac{(2 k-1) !}{[(k-1) !]^{2}}=\frac{1}{2} \frac{(2 k) !}{(k-1) ! k !}$. Consequently, the function $\mathcal{F}_{k, \eta_{k}}(x)$ is completely monotonic on $(0, \infty)$ only if $\eta_{k} \geq \frac{1}{2} \frac{(2 k) !}{(k-1) ! k !}$, while $-\mathcal{F}_{k, \eta_{k}}(x)$ is completely monotonic on $(0, \infty)$ only if $\eta_{k} \leq 0$.

Direct computation gives

$$
\mathfrak{F}_{k, \vartheta_{k}}^{\prime}(x)=(-1)^{k+1} \frac{\psi^{(2 k+1)}(x) \psi^{(k)}(x)-\vartheta_{k} \psi^{(2 k)}(x) \psi^{(k+1)}(x)}{\left[(-1)^{k+1} \psi^{(k)}(x)\right]^{\vartheta_{k}+1}} .
$$

Taking $p=2 k+1, q=k, m=2 k$, and $n=k+1$ in Theorem 3.1 yields that the function

$$
(-1)^{k+1}\left[\psi^{(2 k)}(x) \psi^{(k+1)}(x)-c_{2 k+1,2 k, k+1, k} \psi^{(2 k+1)}(x) \psi^{(k)}(x)\right]
$$

and its negativity are completely monotonic on $(0, \infty)$ if and only if

$$
c_{2 k+1,2 k, k+1, k} \leq \frac{(2 k-1) ! k !}{(2 k) !(k-1) !}=\frac{1}{2}
$$

and

$$
c_{2 k+1,2 k, k+1, k} \geq \frac{(2 k) !(k+1) !}{(2 k+1) ! k !}=\frac{k+1}{2 k+1}
$$

respectively. Therefore, when $\vartheta_{k} \geq 2$, the derivative $\mathfrak{F}_{k, \vartheta_{k}}^{\prime}(x) \leq 0$ on $(0, \infty)$; when $\vartheta_{k} \leq \frac{2 k+1}{k+1}$, the derivative $\mathfrak{F}_{k, \vartheta_{k}}^{\prime}(x) \geq 0$ on $(0, \infty)$. For completeness, in what follows, we will prove these sufficient conditions in details once again.

If $\mathfrak{F}_{k, \vartheta_{k}}^{\prime}(x) \leq 0$, then

$$
\begin{aligned}
\vartheta_{k} & \geq \frac{\psi^{(2 k+1)}(x) \psi^{(k)}(x)}{\psi^{(2 k)}(x) \psi^{(k+1)}(x)} \\
& =\frac{\left[x^{2 k+1} \psi^{(2 k+1)}(x)\right]\left[x^{k} \psi^{(k)}(x)\right]}{\left[x^{2 k} \psi^{(2 k)}(x)\right]\left[x^{k+1} \psi^{(k+1)}(x)\right]} \\
& \rightarrow \frac{(-1)^{2 k}(2 k) !(-1)^{k-1}(k-1) !}{(-1)^{2 k-1}(2 k-1) !(-1)^{k} k !}, \quad x \rightarrow \infty \\
& =2,
\end{aligned}
$$

where we used the limit (2.2).

If $\mathfrak{F}_{k, \vartheta_{k}}^{\prime}(x) \geq 0$, then

$$
\vartheta_{k} \leq \frac{\psi^{(2 k+1)}(x) \psi^{(k)}(x)}{\psi^{(2 k)}(x) \psi^{(k+1)}(x)}
$$




$$
\begin{aligned}
& =\frac{\left[x^{2 k+2} \psi^{(2 k+1)}(x)\right]\left[x^{k+1} \psi^{(k)}(x)\right]}{\left[x^{2 k+1} \psi^{(2 k)}(x)\right]\left[x^{k+2} \psi^{(k+1)}(x)\right]} \\
& \rightarrow \frac{(-1)^{2 k+2}(2 k+1) !(-1)^{k+1} k !}{(-1)^{2 k+1}(2 k) !(-1)^{k+2}(k+1) !}, \quad x \rightarrow 0^{+} \\
& =\frac{2 k+1}{k+1},
\end{aligned}
$$

where we used the limit (2.1).

By the integral representation (3.4), we acquire

$$
\begin{aligned}
\frac{\psi^{(2 k+1)}(x) \psi^{(k)}(x)}{\psi^{(2 k)}(x) \psi^{(k+1)}(x)} & =\frac{\int_{0}^{\infty} \frac{t^{2 k+1}}{1-e^{-t}} e^{-x t} \mathrm{~d} t \int_{0}^{\infty} \frac{t^{k}}{1-e^{-t}} e^{-x t} \mathrm{~d} t}{\int_{0}^{\infty} \frac{t^{2 k}}{1-e^{-t}} e^{-x t} \mathrm{~d} t \int_{0}^{\infty} \frac{t^{k+1}}{1-e^{-t}} e^{-x t} \mathrm{~d} t} \\
& =\frac{\int_{0}^{\infty} t^{2 k} g(t) e^{-x t} \mathrm{~d} t \int_{0}^{\infty} t^{k-1} g(t) e^{-x t} \mathrm{~d} t}{\int_{0}^{\infty} t^{2 k-1} g(t) e^{-x t} \mathrm{~d} t \int_{0}^{\infty} t^{k} g(t) e^{-x t} \mathrm{~d} t} \\
& =\frac{\int_{0}^{\infty}\left[\int_{0}^{t} u^{2 k}(t-u)^{k-1} g(u) g(t-u) \mathrm{d} u\right] e^{-x t} \mathrm{~d} t}{\int_{0}^{\infty}\left[\int_{0}^{t} u^{2 k-1}(t-u)^{k} g(u) g(t-u) \mathrm{d} u\right] e^{-x t} \mathrm{~d} t}
\end{aligned}
$$

By changing the variable $u=\frac{(1+v) t}{2}$, we have

$$
\begin{aligned}
& \frac{\int_{0}^{t} u^{2 k}(t-u)^{k-1} g(u) g(t-u) \mathrm{d} u}{\int_{0}^{t} u^{2 k-1}(t-u)^{k} g(u) g(t-u) \mathrm{d} u} \\
= & \frac{\int_{0}^{1}\left[(1+v)^{k+1}+(1-v)^{k+1}\right]\left(1-v^{2}\right)^{k-1} g\left(\frac{1+v}{2} t\right) g\left(\frac{1-v}{2} t\right) \mathrm{d} v}{\int_{0}^{1}\left[(1+v)^{k-1}+(1-v)^{k-1}\right]\left(1-v^{2}\right)^{k} g\left(\frac{1+v}{2} t\right) g\left(\frac{1-v}{2} t\right) \mathrm{d} v} \\
\rightarrow & \frac{\int_{0}^{1}\left[(1+v)^{k+1}+(1-v)^{k+1}\right]\left(1-v^{2}\right)^{k-1} \mathrm{~d} v}{\int_{0}^{1}\left[(1+v)^{k-1}+(1-v)^{k-1}\right]\left(1-v^{2}\right)^{k} \mathrm{~d} v} \\
= & \frac{2^{3 k} B(2 k+1, k)}{2^{3 k} B(2 k, k+1)} \\
= & 2
\end{aligned}
$$

as $t \rightarrow 0^{+}$, where we used the formula

$$
\begin{aligned}
\int_{0}^{1}\left[(1+x)^{\mu-1}(1-x)^{\nu-1}+(1+x)^{\nu-1}(1-x)^{\mu-1}\right] \mathrm{d} x & =2^{\mu+\nu-1} B(\mu, \nu) \\
& =2^{\mu+\nu-1} \frac{\Gamma(\mu) \Gamma(\nu)}{\Gamma(\mu+\nu)}
\end{aligned}
$$

for $\Re(\mu), \Re(\nu)>0$ in [4, p. 321, 3.214]. Let

$$
\begin{aligned}
& S_{k}(t)= \int_{0}^{1}\left[(1+v)^{k-1}+(1-v)^{k-1}\right]\left(1-v^{2}\right)^{k} g\left(\frac{1+v}{2} t\right) g\left(\frac{1-v}{2} t\right) \mathrm{d} v \\
&-\frac{1}{2} \int_{0}^{1}\left[(1+v)^{k+1}+(1-v)^{k+1}\right]\left(1-v^{2}\right)^{k-1} g\left(\frac{1+v}{2} t\right) g\left(\frac{1-v}{2} t\right) \mathrm{d} v \\
&=\int_{0}^{1} T_{k-1}(v)\left[(1+v)^{k-1}+(1-v)^{k-1}\right]\left(1-v^{2}\right)^{k-1} g\left(\frac{1+v}{2} t\right) g\left(\frac{1-v}{2} t\right) \mathrm{d} v,
\end{aligned}
$$

where

$$
T_{k}(v)= \begin{cases}1-v^{2}-\frac{1}{2} \frac{(1-v)^{k+2}+(1+v)^{k+2}}{(1-v)^{k}+(1+v)^{k}}, & k \in \mathbb{N} \\ \frac{1-3 v^{2}}{2}, & k=0\end{cases}
$$


with $T_{k}(0)=\frac{1}{2}$ for $k \in\{0\} \cup \mathbb{N}, T_{k}(1)=-2$ for $k \in \mathbb{N}$, and $T_{0}(1)=-1$. Combining this with Lemma 2.6. we reveal that $T_{k}(v)$ for $k \in\{0\} \cup \mathbb{N}$ is decreasing on $[0,1]$ and has only one zero $v_{0} \in(0,1)$. Employing the fourth conclusion in Lemma 2.3 yields

$$
\begin{aligned}
& S_{k}(t)=\int_{0}^{v_{0}} T_{k-1}(v)\left[(1+v)^{k-1}+(1-v)^{k-1}\right]\left(1-v^{2}\right)^{k-1} g\left(\frac{1+v}{2} t\right) g\left(\frac{1-v}{2} t\right) \mathrm{d} v \\
& \quad+\int_{v_{0}}^{1} T_{k-1}(v)\left[(1+v)^{k-1}+(1-v)^{k-1}\right]\left(1-v^{2}\right)^{k-1} g\left(\frac{1+v}{2} t\right) g\left(\frac{1-v}{2} t\right) \mathrm{d} v \\
& \geq g\left(\frac{1+v_{0}}{2} t\right) g\left(\frac{1-v_{0}}{2} t\right) \int_{0}^{v_{0}} T_{k-1}(v)\left[(1+v)^{k-1}+(1-v)^{k-1}\right]\left(1-v^{2}\right)^{k-1} \mathrm{~d} v \\
& \quad+g\left(\frac{1+v_{0}}{2} t\right) g\left(\frac{1-v_{0}}{2} t\right) \int_{v_{0}}^{1} T_{k-1}(v)\left[(1+v)^{k-1}+(1-v)^{k-1}\right]\left(1-v^{2}\right)^{k-1} \mathrm{~d} v \\
& \quad=g\left(\frac{1+v_{0}}{2} t\right) g\left(\frac{1-v_{0}}{2} t\right) \int_{0}^{1} T_{k-1}(v)\left[(1+v)^{k-1}+(1-v)^{k-1}\right]\left(1-v^{2}\right)^{k-1} \mathrm{~d} v \\
& =0
\end{aligned}
$$

where, by the formula 3.5 ,

$$
\begin{aligned}
& \int_{0}^{1} T_{k-1}(v)\left[(1+v)^{k-1}+(1-v)^{k-1}\right]\left(1-v^{2}\right)^{k-1} \mathrm{~d} v \\
= & \begin{cases}\int_{0}^{1} \frac{1-3 v^{2}}{2} \mathrm{~d} v, & k=1 \\
\int_{0}^{1}\left[(1+v)^{k-1}+(1-v)^{k-1}\right]\left(1-v^{2}\right)^{k} \mathrm{~d} v & \\
-\frac{1}{2} \int_{0}^{1}\left[(1-v)^{k+1}+(1+v)^{k+1}\right]\left(1-v^{2}\right)^{k-1} \mathrm{~d} v, & k \geq 2\end{cases} \\
= & \begin{cases}0, & k=1 \\
2^{3 k} B(2 k, k+1)-2^{3 k-1} B(2 k+1, k), & k \geq 2\end{cases} \\
= & 0
\end{aligned}
$$

for $k \in \mathbb{N}$. This means that

$$
\frac{\int_{0}^{t} u^{2 k}(t-u)^{k-1} g(u) g(t-u) \mathrm{d} u}{\int_{0}^{t} u^{2 k-1}(t-u)^{k} g(u) g(t-u) \mathrm{d} u}<2 .
$$

on $(0, \infty)$ for $k \in \mathbb{N}$. Accordingly, if $\vartheta_{k} \geq 2$,

$$
\begin{aligned}
& (-1)^{k+1}\left[\vartheta_{k} \psi^{(2 k)}(x) \psi^{(k+1)}(x)-\psi^{(2 k+1)}(x) \psi^{(k)}(x)\right] \\
& =\vartheta_{k} \int_{0}^{\infty}\left[\int_{0}^{t} u^{2 k-1}(t-u)^{k} g(u) g(t-u) \mathrm{d} u\right] e^{-x t} \mathrm{~d} t \\
& -\int_{0}^{\infty}\left[\int_{0}^{t} u^{2 k}(t-u)^{k-1} g(u) g(t-u) \mathrm{d} u\right] e^{-x t} \mathrm{~d} t \\
& =\int_{0}^{\infty}\left[\vartheta_{k}-\frac{\int_{0}^{t} u^{2 k}(t-u)^{k-1} g(u) g(t-u) \mathrm{d} u}{\int_{0}^{t} u^{2 k-1}(t-u)^{k} g(u) g(t-u) \mathrm{d} u}\right] \\
& \quad \times\left[\int_{0}^{t} u^{2 k-1}(t-u)^{k} g(u) g(t-u) \mathrm{d} u\right] e^{-x t} \mathrm{~d} t
\end{aligned}
$$

is positive on $(0, \infty)$. In other words, when $\vartheta_{k} \geq 2$, the first derivative $\mathfrak{F}_{k, \vartheta_{k}}^{\prime}(x)$ is negative on $(0, \infty)$. Consequently, when $\vartheta_{k} \geq 2$, the function $\mathfrak{F}_{k, \vartheta_{k}}(x)$ is decreasing on $(0, \infty)$. 
It is not difficult to verify that

$$
\begin{gathered}
{\left[(1+v)^{k+1}+(1-v)^{k+1}\right]\left(1-v^{2}\right)^{k-1}-\left[(1+v)^{k-1}+(1-v)^{k-1}\right]\left(1-v^{2}\right)^{k}} \\
=2 v\left[(1+v)^{k}-(1-v)^{k}\right]\left(1-v^{2}\right)^{k-1}>0
\end{gathered}
$$

for $v \in(0, \infty)$ and $k \in \mathbb{N}$. This implies that

$$
\frac{\int_{0}^{t} u^{2 k}(t-u)^{k-1} g(u) g(t-u) \mathrm{d} u}{\int_{0}^{t} u^{2 k-1}(t-u)^{k} g(u) g(t-u) \mathrm{d} u} \geq 1
$$

on $(0, \infty)$ for $k \in \mathbb{N}$. Therefore, when $\vartheta_{k} \leq 1<\frac{2 k+1}{k+1}$, the function $\mathfrak{F}_{k, \vartheta_{k}}(x)$ is increasing on $(0, \infty)$.

By Lemma 2.1, we obtain

$$
\begin{aligned}
\lim _{x \rightarrow 0^{+}} \mathfrak{F}_{k, \vartheta_{k}}(x) & =\frac{\lim _{x \rightarrow 0^{+}}\left[x^{2 k+1} \psi^{(2 k)}(x)\right]}{\left(\lim _{x \rightarrow 0^{+}}\left[(-1)^{k+1} x^{k+1} \psi^{(k)}(x)\right]\right)^{\vartheta_{k}}} \lim _{x \rightarrow 0^{+}} x^{(k+1) \vartheta_{k}-(2 k+1)} \\
& =\frac{(-1)^{2 k+1}(2 k) !}{[(k) !]^{\vartheta_{k}}} \lim _{x \rightarrow 0^{+}} x^{(k+1) \vartheta_{k}-(2 k+1)} \\
& = \begin{cases}-\frac{(2 k) !}{[(k) !]^{\frac{2 k+1}{k+1}}}, & \vartheta_{k}=\frac{2 k+1}{k+1} \\
0, & \vartheta_{k}>\frac{2 k+1}{k+1} \\
-\infty, & \vartheta_{k}<\frac{2 k+1}{k+1}\end{cases}
\end{aligned}
$$

and

$$
\begin{aligned}
\lim _{x \rightarrow \infty} \mathfrak{F}_{k, \vartheta_{k}}(x) & =\frac{\lim _{x \rightarrow \infty}\left[x^{2 k} \psi^{(2 k)}(x)\right]}{\left(\lim _{x \rightarrow \infty}\left[(-1)^{k+1} x^{k} \psi^{(k)}(x)\right]\right)^{\vartheta_{k}}} \lim _{x \rightarrow \infty} x^{\left(\vartheta_{k}-2\right) k} \\
& =\frac{(-1)^{2 k-1}(2 k-1) !}{\left[(k-1) ! \vartheta_{k}\right.} \lim _{x \rightarrow \infty} x^{\left(\vartheta_{k}-2\right) k} \\
& = \begin{cases}-\frac{(2 k-1) !}{[(k-1) !]^{2}}, & \vartheta_{k}=2 ; \\
-\infty, & \vartheta_{k}>2 ; \\
0, & \vartheta_{k}<2 .\end{cases}
\end{aligned}
$$

The proof of Theorem 3.2 is complete.

\section{A COMPLETELy MONOTONiC FUNCTION INVOLVING TETRAGAMMA FUNCTION}

For verifying the lower bound in the double inequality (1.3), we establish an upper bound for the third factor in (1.2) and more.

Theorem 4.1. If and only if $\nu \geq 2$, the function

$$
I_{\nu}(x)=\nu\left[\psi^{\prime}(x)-2 \psi^{\prime}(2 x)\right]^{2}-2 \psi^{\prime}(x) \psi^{\prime \prime}(2 x)+\psi^{\prime}(2 x) \psi^{\prime \prime}(x)
$$

is completely monotonic on $(0, \infty)$. Consequently, the double inequality

$$
0<\frac{2 \psi^{\prime}(x) \psi^{\prime \prime}(2 x)-\psi^{\prime}(2 x) \psi^{\prime \prime}(x)}{\left[\psi^{\prime}(x)-2 \psi^{\prime}(2 x)\right]^{2}}<2
$$

is valid on $(0, \infty)$ and sharp in the sense that the lower bound 0 and the upper bound 2 cannot be replaced by any greater number and any less number. 
Proof. Utilizing the duplication formula

$$
\psi(2 z)=\frac{1}{2} \psi(z)+\frac{1}{2} \psi\left(z+\frac{1}{2}\right)+\ln 2
$$

in $[1$, p. 259, 6.3.8] gives

$$
\psi^{\prime}(2 z)=\frac{1}{4}\left[\psi^{\prime}(z)+\psi^{\prime}\left(z+\frac{1}{2}\right)\right]
$$

and

$$
\psi^{\prime \prime}(2 z)=\frac{1}{8}\left[\psi^{\prime \prime}(z)+\psi^{\prime \prime}\left(z+\frac{1}{2}\right)\right] \text {. }
$$

Then

$$
4 I_{\nu}(x)=\nu\left[\psi^{\prime}(x)-\psi^{\prime}\left(x+\frac{1}{2}\right)\right]^{2}-\psi^{\prime}(x) \psi^{\prime \prime}\left(x+\frac{1}{2}\right)+\psi^{\prime}\left(x+\frac{1}{2}\right) \psi^{\prime \prime}(x) .
$$

By the integral representation (3.4) and Lemma 2.2, we obtain

$$
\begin{aligned}
4 I_{\nu}(x)= & \nu\left[\int_{0}^{\infty} g(t)\left(1-e^{-t / 2}\right) e^{-x t} \mathrm{~d} t\right]^{2}-\int_{0}^{\infty} g(t) e^{-x t} \mathrm{~d} t \int_{0}^{\infty} t e^{-t / 2} g(t) e^{-x t} \mathrm{~d} t \\
& +\int_{0}^{\infty} e^{-t / 2} g(t) e^{-x t} \mathrm{~d} t \int_{0}^{\infty} t g(t) e^{-x t} \mathrm{~d} t \\
= & \nu \int_{0}^{\infty}\left[\int_{0}^{t} g(u)\left(1-e^{-u / 2}\right) g(t-u)\left[1-e^{-(t-u) / 2}\right] \mathrm{d} u\right] e^{-x t} \mathrm{~d} t \\
& -\int_{0}^{\infty}\left[\int_{0}^{t} g(u)(t-u) e^{-(t-u) / 2} g(t-u) \mathrm{d} u\right] e^{-x t} \mathrm{~d} t \\
& +\int_{0}^{\infty}\left[\int_{0}^{t} e^{-u / 2} g(u)(t-u) g(t-u) \mathrm{d} u\right] e^{-x t} \mathrm{~d} t \\
= & \int_{0}^{\infty}\left[\int_{0}^{t}\left(\nu-\frac{\left[e^{-(t-u) / 2}-e^{-u / 2}\right](t-u)}{\left(1-e^{-u / 2}\right)\left[1-e^{-(t-u) / 2}\right]}\right)\right. \\
& \left.\times\left(1-e^{-u / 2}\right)\left[1-e^{-(t-u) / 2}\right] g(u) g(t-u) \mathrm{d} u\right] e^{-x t} \mathrm{~d} t .
\end{aligned}
$$

Employing Lemma 2.7 and the positivity of $g(t)$ yields that, when $\nu \geq 2$, the function $4 I_{\nu}(x)$ is completely monotonic on $(0, \infty)$.

By (4.4), if $I_{\nu}(x)$ is completely monotonic on $(0, \infty)$, then its first derivative is

$$
\begin{aligned}
4 I_{\nu}^{\prime}(x)= & 2 \nu\left[\psi^{\prime}(x)-\psi^{\prime}\left(x+\frac{1}{2}\right)\right]\left[\psi^{\prime \prime}(x)-\psi^{\prime \prime}\left(x+\frac{1}{2}\right)\right] \\
& -\psi^{\prime}(x) \psi^{\prime \prime \prime}\left(x+\frac{1}{2}\right)+\psi^{\prime}\left(x+\frac{1}{2}\right) \psi^{\prime \prime \prime}(x) \\
\leq & 0
\end{aligned}
$$

which is equivalent to

$$
\begin{aligned}
\nu & \geq \frac{1}{2} \frac{\psi^{\prime}(x) \psi^{\prime \prime \prime}\left(x+\frac{1}{2}\right)-\psi^{\prime}\left(x+\frac{1}{2}\right) \psi^{\prime \prime \prime}(x)}{\left[\psi^{\prime}(x)-\psi^{\prime}\left(x+\frac{1}{2}\right)\right]\left[\psi^{\prime \prime}(x)-\psi^{\prime \prime}\left(x+\frac{1}{2}\right)\right]} \\
& =\frac{1}{2} \frac{x^{5}\left[\psi^{\prime}(x) \psi^{\prime \prime \prime}\left(x+\frac{1}{2}\right)-\psi^{\prime}\left(x+\frac{1}{2}\right) \psi^{\prime \prime \prime}(x)\right]}{\left(x^{2}\left[\psi^{\prime}(x)-\psi^{\prime}\left(x+\frac{1}{2}\right)\right]\right)\left(x^{3}\left[\psi^{\prime \prime}(x)-\psi^{\prime \prime}\left(x+\frac{1}{2}\right)\right]\right)} \\
& \rightarrow \frac{1}{2} \frac{(-1)^{1+3}(1-1) !(3-1) !(1-3) \frac{1}{2}}{\left[(-1)^{1} 1 ! \frac{1}{2}\right]\left[(-1)^{2} 2 ! \frac{1}{2}\right]} \quad x \rightarrow \infty \\
& =2,
\end{aligned}
$$

where we used the limits $(2.6)$ and 2.8 in Lemma 2.8. 
Since $K(x, y)<0$ was proved in [7, Proposition 5], by the express (1.2), the lower bound in (4.1) is immediate. The upper bound of (4.1) comes from the complete monotonicity of the function $I_{\nu}(x)$. The sharpness of the double inequality (4.1) can be deduced from the limits

$$
\begin{aligned}
& \lim _{x \rightarrow 0^{+}} \frac{2 \psi^{\prime}(x) \psi^{\prime \prime}(2 x)-\psi^{\prime}(2 x) \psi^{\prime \prime}(x)}{\left[\psi^{\prime}(x)-2 \psi^{\prime}(2 x)\right]^{2}} \\
= & \lim _{x \rightarrow 0^{+}} \frac{\psi^{\prime}(x) \psi^{\prime \prime}\left(x+\frac{1}{2}\right)-\psi^{\prime}\left(x+\frac{1}{2}\right) \psi^{\prime \prime}(x)}{\left[\psi^{\prime}(x)-\psi^{\prime}\left(x+\frac{1}{2}\right)\right]^{2}} \\
= & \lim _{x \rightarrow 0^{+}} \frac{x\left[x^{2} \psi^{\prime}(x)\right] \psi^{\prime \prime}\left(x+\frac{1}{2}\right)-\psi^{\prime}\left(x+\frac{1}{2}\right)\left[x^{3} \psi^{\prime \prime}(x)\right]}{\left[x^{2} \psi^{\prime}(x)-x^{2} \psi^{\prime}\left(x+\frac{1}{2}\right)\right]^{2}} \lim _{x \rightarrow 0^{+}} x \\
= & \frac{0(-1)^{2} 1 ! \psi^{\prime \prime}\left(\frac{1}{2}\right)-\psi^{\prime}\left(\frac{1}{2}\right)(-1)^{3} 2 !}{\left[(-1)^{2} 1 !-0\right]^{2}} 0 \\
= & 0
\end{aligned}
$$

and

$$
\begin{aligned}
& \lim _{x \rightarrow \infty} \frac{2 \psi^{\prime}(x) \psi^{\prime \prime}(2 x)-\psi^{\prime}(2 x) \psi^{\prime \prime}(x)}{\left[\psi^{\prime}(x)-2 \psi^{\prime}(2 x)\right]^{2}} \\
= & \lim _{x \rightarrow \infty} \frac{\psi^{\prime}(x) \psi^{\prime \prime}\left(x+\frac{1}{2}\right)-\psi^{\prime}\left(x+\frac{1}{2}\right) \psi^{\prime \prime}(x)}{\left[\psi^{\prime}(x)-\psi^{\prime}\left(x+\frac{1}{2}\right)\right]^{2}} \\
= & \lim _{x \rightarrow \infty} \frac{x^{4}\left[\psi^{\prime}(x) \psi^{\prime \prime}\left(x+\frac{1}{2}\right)-\psi^{\prime}\left(x+\frac{1}{2}\right) \psi^{\prime \prime}(x)\right]}{x^{4}\left[\psi^{\prime}(x)-\psi^{\prime}\left(x+\frac{1}{2}\right)\right]^{2}} \\
= & \frac{(-1)^{1+2}(1-1) !(2-1) !(1-2) \frac{1}{2}}{\left[-(-1)^{1} 1 ! \frac{1}{2}\right]^{2}} \\
= & 2,
\end{aligned}
$$

where we used the formulas 4.2 and (4.3), the limit (2.1) in Lemma 2.1, and the limits (2.6) and (2.8) in Lemma 2.8. The proof of Theorem 4.1 is complete.

\section{The LOWER BOUND OF SECTIONAL CURVATURE}

In this section, we prove the double inequality (1.3) and its sharpness.

Theorem 5.1. For $x>0$, the double inequality $0>\mathcal{K}(x)>-\frac{1}{2}$ is valid on $(0, \infty)$ and sharp in the sense that the lower bound $-\frac{1}{2}$ and the upper bound 0 cannot be replaced by any larger scalar and any smaller scalar respectively.

Proof. By the double inequality (3.3) for $k=1$ in Theorem 3.2, we obtain

$$
-1<\frac{\psi^{\prime \prime}(x)}{\left[\psi^{\prime}(x)\right]^{2}}<0
$$

on $(0, \infty)$. Combining this double inequality with the double inequality (4.1) gives

$$
-2<\frac{\psi^{\prime \prime}(x)}{\left[\psi^{\prime}(x)\right]^{2}} \frac{2 \psi^{\prime}(x) \psi^{\prime \prime}(2 x)-\psi^{\prime}(2 x) \psi^{\prime \prime}(x)}{\left[\psi^{\prime}(x)-2 \psi^{\prime}(2 x)\right]^{2}}<0
$$

which is equivalent to

$$
-\frac{1}{2}<\frac{1}{4} \frac{\psi^{\prime \prime}(x)}{\left[\psi^{\prime}(x)\right]^{2}} \frac{2 \psi^{\prime}(x) \psi^{\prime \prime}(2 x)-\psi^{\prime}(2 x) \psi^{\prime \prime}(x)}{\left[\psi^{\prime}(x)-2 \psi^{\prime}(2 x)\right]^{2}}=\mathcal{K}(x)<0
$$

on $(0, \infty)$, where we used the expression $(1.2)$ for $\mathcal{K}(x)$.

By the limit (2.1), we obtain

$$
\lim _{x \rightarrow 0^{+}} \mathcal{K}(x)=\frac{1}{4} \lim _{x \rightarrow 0^{+}} \frac{\left[x^{3} \psi^{\prime \prime}(x)\right]\left(\left[x^{2} \psi^{\prime}(x)\right]\left[(2 x)^{3} \psi^{\prime \prime}(2 x)\right]-\left[(2 x)^{2} \psi^{\prime}(2 x)\right]\left[x^{3} \psi^{\prime \prime}(x)\right]\right)}{\left[x^{2} \psi^{\prime}(x)\right]^{2}\left[x^{2} \psi^{\prime}(x)-(2 x)^{2} \psi^{\prime}(2 x) / 2\right]^{2}}
$$



$=0$.

By the limit (2.2), we obtain

$$
\begin{aligned}
\lim _{x \rightarrow \infty} \mathcal{K}(x) & =\frac{1}{4} \lim _{x \rightarrow \infty} \frac{x^{2} \psi^{\prime \prime}(x)}{\left[x \psi^{\prime}(x)\right]^{2}} \lim _{x \rightarrow \infty} \frac{x^{4}\left[2 \psi^{\prime}(x) \psi^{\prime \prime}(2 x)-\psi^{\prime}(2 x) \psi^{\prime \prime}(x)\right]}{\left(x^{2}\left[\psi^{\prime}(x)-2 \psi^{\prime}(2 x)\right]\right)^{2}} \\
& =\frac{1}{4} \lim _{x \rightarrow \infty} \frac{x^{4}\left[2 \psi^{\prime}(x) \psi^{\prime \prime}(2 x)-\psi^{\prime}(2 x) \psi^{\prime \prime}(x)\right]}{\left(x^{2}\left[\psi^{\prime}(x)-2 \psi^{\prime}(2 x)\right]\right)^{2}} \\
& =-\frac{1}{2}
\end{aligned}
$$

where, by the second limit in 2.5,

$$
\begin{gathered}
x^{2}\left[\psi^{\prime}(x)-2 \psi^{\prime}(2 x)\right]=x^{2}[\psi(x)-\psi(2 x)]^{\prime}=x^{2}\left[\int_{2 x}^{x} \psi^{\prime}(u) \mathrm{d} u\right]^{\prime} \\
=x^{2}\left[\int_{2}^{1} x \psi^{\prime}(x v) \mathrm{d} v\right]^{\prime}=x^{2} \int_{2}^{1}\left[\psi^{\prime}(x v)+x v \psi^{\prime \prime}(x v)\right] \mathrm{d} v \\
=x^{2} \int_{2}^{1} \Phi^{\prime}(x v) \mathrm{d} v=\int_{2}^{1} \frac{1}{v^{2}}(x v)^{2} \Phi^{\prime}(x v) \mathrm{d} v \\
\rightarrow \int_{2}^{1} \frac{1}{v^{2}} \lim _{x v \rightarrow \infty}\left[(x v)^{2} \Phi^{\prime}(x v)\right] \mathrm{d} v=-\frac{1}{2} \int_{2}^{1} \frac{1}{v^{2}} \mathrm{~d} v=\frac{1}{4}
\end{gathered}
$$

and, by the formulas (4.2) and (4.3) and by the limits (2.2) and (2.7),

$$
\begin{aligned}
& x^{4}\left[2 \psi^{\prime}(x) \psi^{\prime \prime}(2 x)-\psi^{\prime}(2 x) \psi^{\prime \prime}(x)\right] \\
= & \frac{x^{4}}{4}\left[\psi^{\prime}(x) \psi^{\prime \prime}\left(x+\frac{1}{2}\right)-\psi^{\prime}\left(x+\frac{1}{2}\right) \psi^{\prime \prime}(x)\right] \\
= & \frac{x^{4}}{4} \psi^{\prime}(x) \psi^{\prime}\left(x+\frac{1}{2}\right)\left[\frac{\psi^{\prime \prime}\left(x+\frac{1}{2}\right)}{\psi^{\prime}\left(x+\frac{1}{2}\right)}-\frac{\psi^{\prime \prime}(x)}{\psi^{\prime}(x)}\right] \\
= & \frac{x^{4}}{4} \psi^{\prime}(x) \psi^{\prime}\left(x+\frac{1}{2}\right) \int_{x}^{x+1 / 2}\left[\frac{\psi^{\prime \prime}(u)}{\psi^{\prime}(u)}\right]^{\prime} \mathrm{d} u \\
= & \frac{x^{4}}{4} \psi^{\prime}(x) \psi^{\prime}\left(x+\frac{1}{2}\right) \int_{0}^{1 / 2}\left[\frac{\psi^{\prime \prime}(x+u)}{\psi^{\prime}(x+u)}\right]^{\prime} \mathrm{d} u \\
= & \frac{x^{4}}{4} \psi^{\prime}(x) \psi^{\prime}\left(x+\frac{1}{2}\right) \int_{0}^{1 / 2} \frac{\psi^{\prime \prime \prime}(x+u) \psi^{\prime}(x+u)-\left[\psi^{\prime \prime}(x+u)\right]^{2}}{\left[\psi^{\prime}(x+u)\right]^{2}} \mathrm{~d} u \\
= & \frac{1}{4}\left[x \psi^{\prime}(x)\right]\left[\left(x+\frac{1}{2}\right) \psi^{\prime}\left(x+\frac{1}{2}\right)\right] \\
& \times \int_{0}^{1 / 2} \frac{(x+u)^{4}\left(\psi^{\prime}(x+u) \psi^{\prime \prime \prime}(x+u)-\left[\psi^{\prime \prime}(x+u)\right]^{2}\right)}{\left[(x+u) \psi^{\prime}(x+u)\right]^{2}} \frac{x^{3}}{(x+u)^{2}\left(x+\frac{1}{2}\right)} \mathrm{d} u \\
\rightarrow & \frac{1}{4}\left[(-1)^{1-1}(1-1) !\right]^{2} \int_{0}^{1 / 2} \frac{(-1)^{1+2}(1-1) !(2-1) !(1-2)}{\left[(-1)^{1-1}(1-1) !\right]^{2}} \mathrm{~d} u \\
= & \frac{1}{8} \\
& \frac{1}{2} \cdot{ }^{1}(x)
\end{aligned}
$$

as $x \rightarrow \infty$. The proof of Theorem 5.1 is complete.

\section{Several Remarks, Guesses, And open problems}

Finally, we list several remarks, guesses, and open problems related to monotonicity, complete monotonicity, and inequalities of several functions involving polygamma functions. 
Remark 6.1. Theorem 3.1 has been generalized to divided cases in the papers [2, 23].

Remark 6.2. Basing on the double inequalities (3.1) and (3.2), we guess that,

(1) for $m, n \in \mathbb{N}$, the function

$$
Q_{m, n}(x)=\frac{\psi^{(m+n)}(x)}{\psi^{(m)}(x) \psi^{(n)}(x)}
$$

should be decreasing on $(0, \infty)$;

(2) for $m, n, p, q \in \mathbb{N}$ such that $p>m \geq n>q$ and $m+n=p+q$, the function

$$
\mathcal{Q}_{m, n}(x)=\frac{\psi^{(m)}(x) \psi^{(n)}(x)}{\psi^{(p)}(x) \psi^{(q)}(x)}
$$

should be decreasing on $(0, \infty)$.

Remark 6.3. The necessary and sufficient condition $\eta_{1} \geq 1$ in Theorem 3.2 for the function $\mathcal{F}_{1, \eta_{1}}(x)$ to be completely monotonic on $(0, \infty)$ has been established in [20, Theorem 3]. For more information on results related to the function $\mathcal{F}_{1, \eta_{1}}(x)$, please refer to the papers $[11,19]$, the review and survey articles [12, 17], and a number of closely related references therein.

Remark 6.4. Motivated by Theorem 3.2 in this paper, [6, Theorem 1], and the fifth and sixth problems in [17, Sections 12.5 and 12.6], we pose a new and more reasonable open problem as follows. For $n \in \mathbb{N}$ and $\sigma_{k, m} \in \mathbb{R}$, let

$$
F_{n}(x)=\sum_{\ell m=n} \sigma_{\ell, m}\left[\psi^{(\ell)}(x)\right]^{m} .
$$

It is clear that $\sigma_{1,1} \geq 0$ is a necessary and sufficient condition for the function $F_{1}(x)$ to be completely monotonic on $(0, \infty)$. When $n=2$, since

$$
F_{2}(x)=\sigma_{1,2}\left[\psi^{\prime}(x)\right]^{2}+\sigma_{2,1} \psi^{\prime \prime}(x),
$$

we conclude from [20, Theorem 3] and Theorem 3.2 that both the condition $\sigma_{2,1} \geq$ $\sigma_{1,2}>0$ and the condition $\sigma_{1,2} \geq 0$ and $\sigma_{2,1}<0$ are necessary and sufficient for $F_{2}(x)$ to be completely monotonic on $(0, \infty)$. Theorem 3.2 in this paper means that, when $\sigma_{\ell, m}=0$ for all $(\ell, m) \notin\{(2 k, 1),(k, 2)\}$, the function $F_{2 k}(x)$ for $k \in \mathbb{N}$ is completely monotonic on $(0, \infty)$ if and only if $\sigma_{2 k, 1}<0$ and $\sigma_{k, 2} \geq 0$, or if and only if $\sigma_{2 k, 1}>0$ and $\frac{\sigma_{k, 2}}{\sigma_{2 k, 1}} \geq \frac{1}{2} \frac{(2 k) !}{(k-1) ! k !}$.

When $n \geq 3$, what are the necessary and sufficient conditions on $\sigma_{k, m}$ such that the function $F_{n}(x)$ is completely monotonic on $(0, \infty)$ ?

Remark 6.5. For $\ell, m \in \mathbb{N}$ with $\ell \neq m$, real numbers $b>0$ and $c \geq 0$ with $(b, c) \neq(1,0)$, and $\xi_{\ell, m} \in \mathbb{R}$, find necessary and sufficient condition on $\bar{\xi}_{\ell, m}$ such that the function

$$
\psi^{(\ell)}(x) \psi^{(m)}(b x+c)-\xi_{\ell, m} \psi^{(m)}(x) \psi^{(\ell)}(b x+c)
$$

is completely monotonic in $x \in(0, \infty)$.

Remark 6.6. Motivated by Theorem 4.1, we guess that the functions

$$
\frac{2 \psi^{\prime}(x) \psi^{\prime \prime}(2 x)-\psi^{\prime}(2 x) \psi^{\prime \prime}(x)}{\left[\psi^{\prime}(x)-2 \psi^{\prime}(2 x)\right]^{2}}=\frac{\psi^{\prime}(x) \psi^{\prime \prime}\left(x+\frac{1}{2}\right)-\psi^{\prime}\left(x+\frac{1}{2}\right) \psi^{\prime \prime}(x)}{\left[\psi^{\prime}(x)-\psi^{\prime}\left(x+\frac{1}{2}\right)\right]^{2}}
$$

and

$$
\begin{aligned}
Y(x) & =\frac{4 \psi^{\prime}(x) \psi^{\prime \prime \prime}(2 x)-\psi^{\prime}(2 x) \psi^{\prime \prime \prime}(x)}{\left[\psi^{\prime}(x)-2 \psi^{\prime}(2 x)\right]\left[\psi^{\prime \prime}(x)-4 \psi^{\prime \prime}(2 x)\right]} \\
& =\frac{\psi^{\prime}(x) \psi^{\prime \prime \prime}\left(x+\frac{1}{2}\right)-\psi^{\prime}\left(x+\frac{1}{2}\right) \psi^{\prime \prime \prime}(x)}{\left[\psi^{\prime}(x)-\psi^{\prime}\left(x+\frac{1}{2}\right)\right]\left[\psi^{\prime \prime}(x)-\psi^{\prime \prime}\left(x+\frac{1}{2}\right)\right]}
\end{aligned}
$$


should be increasing on $(0, \infty)$ and the double inequality $0<Y(x)<4$ should be valid on $(0, \infty)$ and sharp in the sense that the lower bound 0 and the upper bound 4 cannot be replaced by any larger number and any smaller number respectively.

Remark 6.7. This paper is a slightly revised version of the preprint 13 and the fifth one in a series of articles including $[10,14,15,16]$.

\section{REFERENCES}

[1] M. Abramowitz and I. A. Stegun (Eds), Handbook of Mathematical Functions with Formulas, Graphs, and Mathematical Tables, National Bureau of Standards, Applied Mathematics Series 55, Reprint of the 1972 edition, Dover Publications, Inc., New York, 1992.

[2] P. Gao, Some completely monotonic functions involving the polygamma functions, J. Inequal. Appl. 2019, Paper No. 218, 9 pages; available online at https://doi.org/10.1186/ s13660-019-2172-x

[3] P. Gao, Some monotonicity properties of gamma and q-gamma functions, ISRN Math. Anal. 2011, Art. ID 375715, 15 pages; available online at https://doi.org/10.5402/2011/375715

[4] I. S. Gradshteyn and I. M. Ryzhik, Table of Integrals, Series, and Products, Translated from the Russian, Translation edited and with a preface by Daniel Zwillinger and Victor Moll, Eighth edition, Revised from the seventh edition, Elsevier/Academic Press, Amsterdam, 2015; available online at https://doi.org/10.1016/B978-0-12-384933-5.00013-8

[5] B.-N. Guo and F. Qi, A completely monotonic function involving the tri-gamma function and with degree one, Appl. Math. Comput. 218 (2012), no. 19, 9890-9897; available online at https://doi.org/10.1016/j.amc.2012.03.075

[6] B.-N. Guo, F. Qi, and H. M. Srivastava, Some uniqueness results for the non-trivially complete monotonicity of a class of functions involving the polygamma and related functions, Integral Transforms Spec. Funct. 21 (2010), no. 11, 103-111; available online at http://dx.doi.org/10.1080/10652461003748112

[7] A. Le Brigant and S. Puechmorel, The Fisher-Rao geometry of beta distributions applied to the study of canonical moments, arXiv preprint (2019), available online at https://arxiv. org/abs/1904.08247v1.

[8] A. Le Brigant, S. Preston, and S. Puechmorel, Fisher-Rao geometry of Dirichlet distributions, arXiv preprint (2020), available online at https://arxiv.org/abs/2005.05608

[9] D. S. Mitrinović, J. E. Pečarić, and A. M. Fink, Classical and New Inequalities in Analysis, Kluwer Academic Publishers, Dordrecht-Boston-London, 1993; available online at https: //doi.org/10.1007/978-94-017-1043-5

[10] F. Qi, Complete monotonicity and monotonicity of two functions defined by two derivatives of a function involving trigamma function, HAL preprint (2020), available online at https: //hal.archives-ouvertes.fr/hal-02993723

[11] F. Qi, Complete monotonicity of a function involving the tri- and tetra-gamma functions, Proc. Jangjeon Math. Soc. 18 (2015), no. 2, 253-264; available online at https://doi.org/ 10.17777/pjms.2015.18.2.253

[12] F. Qi, Completely monotonic degree of a function involving trigamma and tetragamma functions, AIMS Math. 5 (2020), no. 4, 3391-3407; available online at https://doi.org/10.3934/ math.2020219

[13] F. Qi, Lower bound of sectional curvature of manifold of beta distributions and complete monotonicity of functions involving polygamma functions, MDPI Preprints 2020, 202011????, 8 pages; available online at https://doi.org/10.20944/preprints202011.???? . $\mathrm{v} 1$

[14] F. Qi, Monotonicity of a ratio involving trigamma and tetragamma functions, OSF Preprints (2020), available online at https://doi.org/10.31219/osf.io/5rfb8.

[15] F. Qi, Necessary and sufficient conditions for two functions defined by two derivatives of a function involving trigamma function to be completely monotonic or monotonic, OSF Preprints (2020), available online at https://doi.org/10.31219/osf.io/6ar4p.

[16] F. Qi, Some properties of several functions involving polygamma functions and originating from the sectional curvature of the beta manifold, São Paulo J. Math. Sci. 15 (2021), in press; available online at https://doi.org/10.1007/s40863-020-00193-1

[17] F. Qi and R. P. Agarwal, On complete monotonicity for several classes of functions related to ratios of gamma functions, J. Inequal. Appl. 2019, Paper No. 36, 42 pages; available online at https://doi.org/10.1186/s13660-019-1976-z

[18] F. Qi and C. Berg, Complete monotonicity of a difference between the exponential and trigamma functions and properties related to a modified Bessel function, Mediterr. 
J. Math. 10 (2013), no. 4, 1685-1696; available online at https://doi.org/10.1007/ s00009-013-0272-2

[19] F. Qi and B.-N. Guo, Complete monotonicity of divided differences of the di- and tri-gamma functions with applications, Georgian Math. J. 23 (2016), no. 2, 279-291; available online at https://doi.org/10.1515/gmj-2016-0004

[20] F. Qi and B.-N. Guo, Necessary and sufficient conditions for functions involving the triand tetra-gamma functions to be completely monotonic, Adv. Appl. Math. 44 (2010), no. 1, 71-83; available online at https://doi.org/10.1016/j.aam.2009.03.003

[21] R. L. Schilling, R. Song, and Z. Vondraček, Bernstein Functions, 2nd ed., de Gruyter Studies in Mathematics 37, Walter de Gruyter, Berlin, Germany, 2012; available online at https: //doi.org/10.1515/9783110269338

[22] D. V. Widder, The Laplace Transform, Princeton University Press, Princeton, 1946.

[23] Z.-H. Yang, Some properties of the divided difference of psi and polygamma functions, J. Math. Anal. Appl. 455 (2017), no. 1, 761-777; available online at https://doi.org/10. $1016 / j \cdot j$ maa.2017.05.081 